\title{
Convergências entre prevenção quaternária e promoção da saúde
}

\author{
Convergences between quaternary prevention and health promotion \\ Convergencias entre prevención cuaternaria y promoción de la salud
}

\author{
Charles Dalcanale Tesser ${ }^{1} \odot$ \\ ${ }^{1}$ Universidade Federal de Santa Catarina (UFSC). Florianópolis/SC
}

\section{Resumo}

Introdução: A prevenção quaternária (P4) e a promoção da saúde (PS) vem se desenvolvendo em paralelo. Objetivo: Apresentar e discutir algumas relações de convergência da P4 com a PS. Métodos: Trata-se de ensaio teórico que relaciona ideias e literatura selecionadas assistematicamente, consideradas essenciais para a discussão das relações abordadas. Resultados: A P4 converge com a PS por duas vias: a) pelas suas relações com a medicalização social e b) pelas suas convergências com as ações que Geoffrey Rose chamou de medidas preventivas 'redutivas' - reduzir riscos aumentados de adoecimento reduzindo exposições existentes na vida moderna urbana. A medicalização gerada no cuidado clínico-sanitário reduz a autonomia relativa no manejo de sofrimentos, dores e adoecimentos; e produz dependência excessiva de ações profissionalizadas. O empoderamento individual e comunitário amplia a autonomia, e isso é um objetivo importante da PS. Quanto mais exitosa a PS, menor a medicalização; e quanto maior a medicalização, mais difícil a PS. A P4 converge com a PS por sua inibição da medicalização derivada da ação clínica. A PS visa também impactar determinantes gerais da saúde-doença. Isso, operacionalmente, a aproxima e por vezes a identifica com a prevenção redutiva. A P4 induz preferência relativa pela prevenção redutiva por ser mais segura e intensifica a atitude crítica para com a prevenção 'aditiva' - introdução de fatores protetores artificiais (vacinas, hipolipemiantes, rastreamentos). Assim, a prática da P4 converge operacionalmente com ações de PS. Conclusão: A P4 é convergente com a PS em dimensão microssocial - nos serviços de atenção primária à saúde (APS) - e macrossocial. A compreensão da interface da P4 com a PS facilita sua exploração, realçando a relevância da P4 no cuidado clínico e em ações coletivas na APS.

Palavras-chave: Prevenção Quaternária; Promoção da Saúde; Atenção Primária à Saúde; Sistema Único de Saúde.
Como citar: Tesser CD. Convergências entre prevenção quaternária e promoção da saúde. Rev Bras Med Fam Comunidade. 2020;15(42):2515. https://doi.org/10.5712/rbmfc15(42)2515
Autor correspondente: Charles Dalcanale Tesser E-mail: charles.tesser@ufsc.br Fonte de financiamento: Não se aplica Parecer CEP: Não se aplica Procedência: não encomendado Avaliação por pares: externa Recebido em: 11/05/2020. Aprovado em: 18/08/2020. 


\begin{abstract}
Introduction: Quaternary prevention (P4) and health promotion (PS) are been developing in parallel. Objective: To present and discuss some relations of convergence between P4 and PS. Methods: This is a theoretical essay that relates ideas and literature selected unsystematically, considered essential for the discussion of the relationships addressed. Results: P4 converges with PS in two ways: a) due to its relationship with social medicalization and b) due to its convergence with actions that Geoffrey Rose called 'reductive' preventive measures - reducing increased risks of illness by reducing artificial exposures existing in the urban modern life. The medicalization generated in clinical and health care reduces the relative autonomy in the management of situations of suffering, pain and illness; and produces excessive dependence on professionalized actions. Individual and community empowerment expands autonomy, and this is an important goal of PS. The more successful the PS, the lower the medicalization; and the greater the medicalization, the more difficult the PS. P4 converges with PS due to its inhibition of medicalization derived from clinical action. PS also aims to impact general determinants of health-disease. This, operationally, brings it closer and sometimes identifies it with reductive prevention. P4 induces a relative preference for reductive prevention because it is safer and because it intensifies the critical attitude towards 'additive' prevention - the introduction of artificial protective factors (vaccines, lipid-lowering drugs and screening). Thus, the practice of P4 converges operationally with PS actions. Conclusion: P4 is convergent with PS in a micro-social dimension - in primary health care services $(\mathrm{PHC})$ - and macro-social. Understanding the interface between $\mathrm{P} 4$ and $\mathrm{PS}$ facilitates its exploration, highlighting the relevance of P4 in clinical care and collective actions in PHC.
\end{abstract}

Keywords: Quaternary Prevention; Health Promotion; Primary Health Care; Unified Health System.

\title{
Resumen
}

Introducción: la prevención cuaternaria (P4) y la promoción de la salud (PS) se han desarrollado en paralelo. Objetivo: presentar y discutir algunas relaciones de convergencia entre P4 y PS. Método: Este es un ensayo teórico que relaciona ideas y literatura seleccionada de manera no sistemática, considerada esencial para la discusión de las relaciones abordadas. Resultados: P4 converge con PS de dos maneras: a) por sus relaciones con la medicalización social y b) por su convergencia con acciones que Geoffrey Rose llamó medidas preventivas 'reductoras', que reducen riesgos amplificados de enfermedad al reducir las exposiciones artificiales existentes en la vida urbana moderna. La medicalización generada en la atención clínica y sanitaria reduce la autonomía relativa en el manejo del sufrimiento, el dolor y la enfermedad y produce dependencia excesiva de acciones profesionalizadas. El empoderamiento individual y comunitario amplía la autonomía, y este es un objetivo importante de PS. Cuanto más exitoso es el PS, menor es la medicalización; y cuanto mayor es la medicalización, más difícil es la PS. P4 converge con PS debido a su inhibición de la medicalización derivada de la acción clínica. PS también tiene como objetivo impactar los determinantes generales de la enfermedad de salud. Esto, operativamente, lo acerca y a veces lo identifica con la prevención reductiva. P4 induce una preferencia relativa por la prevención reductiva porque es más segura y porque intensifica la actitud crítica hacia la prevención 'aditiva': la introducción de factores protectores artificiales (vacunas, fármacos hipolipemiantes, tamizajes). Por lo tanto, la práctica de P4 converge operacionalmente con acciones de PS. Conclusión: P4 es convergente con PS en una dimensión microsocial - en los servicios de atención primaria de salud (APS) - y macrosocial. Comprender la interfaz entre P4 y PS facilita su exploración, destacando la relevancia de P4 en la atención clínica y en las acciones colectivas en APS.

Palabras clave: Prevención Cuaternaria; Promoción de la Salud; Atención Primaria de Salud; Sistema Único de Salud.

\section{INTRODUÇÃO}

A prevenção quaternária (P4) foi conceituada pelos médicos de família e comunidade como ações realizadas para identificar um paciente ou população em risco de sobremedicalização, para protegê-los de novas invasões médicas e sugerir-Ihes intervenções que sejam eticamente aceitáveis. ${ }^{1}$ Em contextos diversos outros significados foram dados à $\mathrm{P} 4$, aqui não abordados. ${ }^{2} \mathrm{O}$ conceito foi proposto por Jamoulle, ${ }^{3}$ no final da década de 1980, e lentamente vem se desenvolvendo em vários países. Inicialmente focado nas situações em que o paciente se sente mal, mas não tem doença (medicamente diagnosticável), o conceito se expandiu para o enfrentamento de todas as situações de uso excessivo de biomedicina, ancorado no princípio da não-maleficência.

P4 é um conceito complexo baseado em fundamentos históricos, teóricos e científicos, envolvendo diversas áreas do conhecimento. Uma compreensão mínima desses fundamentos e de suas interconexões é essencial para se desenvolver intervenções eficazes no manejo de procedimentos médicos desnecessários e seus efeitos iatrogênicos. ${ }^{4}$

A Carta de Ottawa ${ }^{5}$ definiu a promoção da saúde (PS) como processo de capacitação da comunidade para atuar na melhoria da sua qualidade de vida e saúde, incluindo uma maior participação no controle 
deste processo. Na PS, além da orientação às pessoas para que adotem estilos de vida saudáveis, há a valorização do papel de um amplo espectro de fatores socioeconômicos e ambientais sobre a saúdedoença, chamados determinantes gerais ou sociais.

A P4 e a PS vêm se desenvolvendo relativamente em paralelo, com pouca correlação entre seus conceitos e práticas, especialmente considerando-se o contexto dos serviços de atenção primária à saúde (APS). A P4 nasceu no ambiente da clínica na APS, em que a dimensão do cuidado clínico individual tem proeminência. Mas também na APS há grande valorização (cada vez maior, devido a maior longevidade e à proeminência das doenças crônicas) para a prevenção de doenças e a promoção da saúde em dimensão individual, voltadas às pessoas, famílias e comunidades. ${ }^{6}$

APS contemporânea, por sua vez, floresceu no ambiente da saúde pública, em que o enfoque é mais coletivo e macrossocial, voltado para ações de maior abrangência, com foco na população toda, incluindo sociedade, economia, política e cultura, indo além do sistema de saúde. ${ }^{7}$ Ou seja, a PS tem como seu foco principal e talvez maior potência a dimensão macrossocial ou societal, embora também haja grande apelo para que permeie o cuidado clínico em dimensão microssocial.

Embora a PS e a P4 tenham desenvolvimentos diferenciais, histórias e potências distintas em relação às dimensões individual e coletiva do processo saúde-doença-cuidado, ambas têm amplo escopo e devem ser praticadas e desenvolvidas tanto para pessoas e famílias quanto para populações inteiras.

\section{OBJETIVO}

O objetivo deste ensaio é apresentar relações da P4 com a PS. Nossa tese é que a P4 tem relações específicas de convergência com a PS, teóricas e práticas, tanto em dimensão individual como coletiva, embora indiretas. Todavia, essas relações nem sempre são visíveis, reconhecidas e compreendidas, motivo pelo qual se justifica esse trabalho.

As relações entre PS e P4 ocorrem por pelo menos duas vias. Uma delas é relação quase direta das duas com o processo de medicalização social, caracterizado pela conversão de situações antes manejadas autonomamente em doenças, transtornos ou riscos abordados profissionalmente. ${ }^{8}$ Tal processo tem certo antagonismo ou proporcionalidade inversa para com a PS e a P4: quanto mais exitosa a PS e a P4, menor a medicalização social derivada do cuidado clínico-sanitário; quanto maior a intensidade e velocidade da medicalização social, maior a necessidade de PS e de P4.

A outra via é a relação da PS e da P4 com as ações preventivas conforme analisadas por Geoffrey Rose, que as categorizou em medidas preventivas 'redutivas' e 'aditivas'. ${ }^{9}$ As medidas preventivas redutivas são as que reduzem riscos de adoecimento aumentados nas sociedades urbanizadas, como: sedentarismo, tabagismo, obesidade, poluição ambiental, multiprocessamento e agrotóxicos nos alimentos, etc., envolvendo geralmente prevenção primária e redução da exposição. Como vários desses riscos são considerados determinantes gerais da saúde-doença, de que trata a PS, sua redução significa convergência operacional entre várias medidas preventivas redutivas e de PS.

Por seu turno, a P4 orienta uma relativa preferência por medidas preventivas redutivas em relação a medidas de prevenção aditiva, caracterizadas pela introdução de fatores artificiais de proteção (vacinas, rastreamentos, hipolemiantes, etc.), sobretudo em estratégia de alto risco, devido ao seu grande potencial iatrogênico e medicalizante e seu pouco impacto na morbimortalidade coletiva. ${ }^{10}$ Isso significa que a prática 
da P4 valoriza e reforça a prevenção redutiva, sobretudo em abordagem populacional, gerando, portanto, outra convergência com a PS.

\section{MÉTODOS}

Do ponto de vista metodológico, trata-se de um ensaio teórico relacionando P4 e PS, a partir de literatura selecionada (assistemática e propositalmente), considerada essencial para as relações tematizadas. A apresentação segue a ordem das duas vias convergentes entre PS e P4 mencionadas, dividida em seis tópicos curtos. É apresentada uma síntese dos conceitos e relações subjacentes às afirmações acima, de modo progressivo e à medida em que a argumentação avança. $O$ ensaio discute relações teóricas, mas também práticas e operacionais, havendo destaque para o contexto da APS, nascedouro e contexto privilegiado da prática de P4, mas também de PS em dimensão individual, familiar e comunitária. O próprio texto indica transições de nível de argumentação ou de contexto de aplicação, em que pese o espaço exíguo ter imposto um tom genérico e teórico, com pouca exemplificação empírica.

\section{RESULTADOS E DISCUSSÃO}

\section{Prevenção quaternária e medicalização}

A P4 tem direta relação com a parte da medicalização social que é gerada no cuidado clínico, a qual é significativa, ao menos no contexto clínico individual e comunitário. Por definição, quanto mais P4, menor medicalização desnecessária e menor medicalização em geral derivadas da ação profissional e institucional. Isso é desejável, mas para o entendimento e a aceitação dessa afirmação é necessário fundamentar porque é desejável reduzir a medicalização em geral decorrente do cuidado clínico, para além de evitar apenas o seu excesso, o que é consensual, especialmente na APS.

O fenômeno da medicalização social, segundo Conrad, ${ }^{8}$ envolve a aplicação dos saberes, técnicas e tecnologias biomédicas a um determinado problema ou situação, quando isso não era feito anteriormente. Geralmente, isso implica interpretar problemas, aspectos ou experiências das pessoas como doenças, transtornos ou riscos, conforme a biomedicina, e tratá-los por meio das suas técnicas consagradas. Inicialmente discutida tendo como referência a biomedicina, com a proliferação de profissões na área da saúde, hoje podemos falar de medicalização em um sentido mais geral, relacionado à aplicação dos saberes e práticas das várias profissões da saúde: poder-se-ia falar de 'psicologização social', 'nutricionização social', etc. Para evitar neologismos, mantemos o termo medicalização, estendendo-o às demais profissões da saúde.

O conceito de Conrad, ${ }^{8}$ é descritivo e não valorativo, nada diz sobre ser a medicalização ruim ou boa. A medicalização pode ser vantajosa em certas situações e desvantajosa em outras. Ela vem ocorrendo há alguns séculos nas sociedades europeias, ${ }^{11}$ e há consenso sobre ser um processo complexo social e cultural em aceleração no século $21 .{ }^{12}$ A medicalização também pode ser desfeita, como no caso da homossexualidade. Em alguns casos, ela pode ser benéfica, como em catástrofes ambientais, quando a abordagem médica pode legitimar socialmente a necessidade de cuidados aos atingidos. ${ }^{13}$

Por outro lado, para Illich, ${ }^{14}$ a medicalização é essencialmente indesejável e deve ser evitada o quanto possível. A razão é que, além de produzir frequentemente iatrogenia clínica, a medicalização tende a reduzir a 
autonomia e a competência de pessoas e coletividades para gerir autonomamente boa parte das situações de sofrimento, adoecimento, dor, nascimento e morte. Nesse sentido, a medicalização geralmente envolve algum prejuízo à saúde, tomada esta última como o grau de liberdade vivida, grau de competência e de autonomia para manejar as oscilações e vicissitudes da vida, segundo Canguilhem. ${ }^{15} \mathrm{Ou}$ seja, na medicalização há embutida uma redução de autonomia, que é relativa e que não deveria ser rebaixada pela ação profissional. E também um aumento ou geração de dependência para com a ação profissional.

A visão crítica de Illich, ${ }^{14}$ valoriza esse componente negativo das consequências ou características indesejáveis da medicalização, que aqui assumimos. AP4, assim, tem o significado geral, salvo exceções, de reduzir o grau de medicalização inerente ao cuidado clínico-sanitário, para além de reduzir os excessos de medicalização que são consensualmente condenáveis e evitáveis. Isso justifica recusar a recente proposta de Martins et al. ${ }^{16}$ de eliminar do conceito de $\mathrm{P} 4$ a referência à medicalização, mantendo apenas a redução de danos iatrogênicos, expressa como busca de melhor balanço benefícios $\mathrm{x}$ danos. O significado da P4 como originalmente proposto é equilibrado ${ }^{17}$ e deve ser mantido.

\section{Medicalização e promoção da saúde}

APS também tem uma relação direta com a medicalização. Mas para entendê-la, convém recuperar sinopticamente os principais significados atribuídos à PS, que mudaram no tempo. O modelo da história natural das doenças, de Leavell e Clark, ${ }^{18}$ concebia a PS como uma parte da prevenção, um tipo inespecífico de prevenção primária (ação realizada antes do aparecimento da doença para evitá-la), vinculado ao cuidado aos indivíduos e famílias, sobretudo por meio de ações educativas. Esse modelo conectava a prevenção com as ações médicas, buscando induzir uma atitude mais preventiva nesses profissionais. ${ }^{19}$ No início do século XXI, a prevenção já tem uma grande penetração na atividade clínica, especialmente via ações preventivas aditivas aplicadas em indivíduos de alto risco. ${ }^{20}$

A Carta de Ottawa, em 1986, ${ }^{5}$ marcou uma renovação do conceito e da formulação de estratégias para a implementação da PS. Ela enfatizou a dimensão social da saúde, ressaltando o processo que possibilita ao indivíduo e sua comunidade aumentarem sua autonomia para maior controle e melhoria da qualidade de vida e de saúde, sendo esta compreendida como um recurso para o bem viver. A Carta de Ottawa refere cinco campos de ação para a PS: políticas públicas saudáveis, ambientes favoráveis à saúde, reforço da ação comunitária, desenvolvimento de habilidades pessoais e reorientação dos serviços de saúde. Os documentos orientadores da PS sugerem a viabilização de espaços na APS que estimulem a produção social da saúde, nos quais vários tipos de grupos podem participar, atribuindo sentidos e dialogando sobre as temáticas e ações propostas. ${ }^{21}$

Há na PS uma valorização da participação social e uma ênfase na abordagem de determinantes gerais do processo saúde-doença por múltiplas vias. Uma dessas vias é a construção de processos de 'empoderamento', termo que vai na direção de maiores níveis de autonomia individual, comunitária e societal na definição de decisões, estratégias de ação individual e coletiva e até intervenções em políticas públicas e sociais, de forma crítica e democrática, valorizando a solidariedade e a saúde. ${ }^{22}$ Isso implica em altos níveis de empoderamento individual e psicológico (maior motivação, senso de coerência e sentimento de pertencimento dos indivíduos), mas também mais disseminação em pessoas e coletividades de capacidade de reflexão crítica e ação na vida pessoal, social e política, o que Carvalho e Gastaldo, em 
$2008,{ }^{23}$ chamaram de empoderamento coletivo. Considerando o empoderamento coletivo como uma das características ou objetivos da PS, salta aos olhos, a uma primeira visada, seu sentido geral inverso ao do processo de medicalização social nos seus aspectos indesejáveis acima referidos.

Todavia, a PS não é um movimento ou um conjunto discursivo homogêneo. Ela pode ser dissecada em quatro dimensões bipolares, ${ }^{24}$ que incluem complementaridades, mas também divergências. Na dimensão da ação, há um polo centrado na ação sobre indivíduos, realizada pelo setor saúde; e outro polo que enfatiza a ação intersetorial centrada em coletividades e sociedades. A terceira dimensão, epistemológica ou conceitual, carrega uma polaridade de concepções: por um lado há uma concepção positiva de saúde, que poderia ser sintetizada, à moda de Canguilhem, ${ }^{15} \mathrm{em}$ termos de saúde como grau de liberdade e autonomia vividas com responsabilidade; e no outro lado vigora uma concepção biomédica que maneja a saúde como ausência ou controle da doença e seus riscos, dado o seu centramento teórico e operacional nas entidades doenças. ${ }^{25}$ Por fim, na quarta dimensão, pedagógica, há, por um lado, uma tradição educativa autoritária, hierárquica e controlista (talvez herdada dos ambientes hospitalares e da relação biomedicinaEstado); e, de outro lado, há uma visão educativa emancipatória centrada no apoio solidário, dialogal e empático em relações horizontalizadas. APS, em uma vertente crítica aqui adotada como referência, busca a superação da tradição autoritária, visando o empoderamento fomentador da autonomia, da participação e da democracia, em sintonia com a concepção de saúde entendida como liberdade vivida.

O processo de medicalização tende a hipertrofiar e estender a abordagem biomédica, com sua forte tradição autoritária, controlista e culpabilizadora. ${ }^{26,27}$ Por outro lado, a geração de dependência produzida pela medicalização tende a dificultar a ampliação da autonomia e da participação. A dependência crescente facilita a projeção de cada vez mais poderes para interpretação e intervenção em situações ou problemas da vida nos profissionais (seus saberes e tecnologias). Com isso, é lógica a tendência de que quanto mais medicalização menor a autonomia e mais difícil a PS e o empoderamento; e, reciprocamente, quanto mais PS menor a medicalização, ou pelo menos mais fácil evitar a medicalização e resistir a ela.

\section{Prevenção quaternária, medicalização e promoção da saúde}

Identificadas as relações acima da PS e da P4 com a medicalização, tornam-se compreensíveis facilmente as relações entre as duas primeiras. Sendo a PS e a medicalização inversamente proporcionais uma à outra ou divergentes e antagônicas entre si, fica fácil considerar a relação da PS com a P4, já que esta última também tem uma relação inversa ou contrária à medicalização, limitando-a, reduzindo-a e evitando danos iatrogênicos.

Ao reduzir as tendências medicalizantes do cuidado médico e profissional, a P4 tende, por consequência, a facilitar a abertura de espaços e a valorização da parceria horizontalizada para diálogos entre profissionais e usuários, famílias e comunidades, de certa forma fomentando indiretamente, porque isso é necessário à consecução da $\mathrm{P} 4$, o compartilhamento de decisões e a relativização crítica do ideário biomédico e suas técnicas, a consideração de seus limites, perigos e danos. A prática da P4 tende a abrir espaço para essas direções, já que requer, para se concretizar, postura dialogal empática e contextualização e compartilhamento de decisões, crucial para a P4 no cuidado clínico aos adoecidos, e mais ainda no cuidado preventivo. ${ }^{19}$ Assim, se não há relação conceitual direta entre P4 e PS, há relação indireta: ela converge com a PS, facilitando-a. 
Isso é importante, porque comumente se orienta fazer PS nos serviços de APS, mas nem sempre são claras quais iniciativas são adequadas, convergentes e coerentes com a PS no ambiente microssocial do cuidado individual e comunitário nesses serviços. A P4 ajuda a vislumbrar como a PS entrelaça-se com o cuidado clínico de boa qualidade, para adoecidos e na prevenção, para além de outras iniciativas de PS.

\section{Prevenção quaternária e prevenção redutiva}

Rose $^{9}$ elaborou uma reflexão crítica sobre a abordagem preventiva em saúde pública e medicina, ao analisar as bases da prevenção. Destacou em sua análise a tendência de ser adotada uma estratégia preventiva por ele chamada 'abordagem de alto risco', que consiste em identificar um grupo de maior risco de adoecimento e nele intervir preventivamente. Isso é que se faz quando tratamos hipertensão arterial, identificamos tabagistas e alcoolistas e oferecemos cuidados para redução ou parada desses hábitos.

Rose $^{9}$ também discutiu o que chamou de abordagem populacional, outra estratégia preventiva, na qual em vez de se atuar sobre pessoas de alto risco se busca reduzir o risco de determinado adoecimento ou agravo na população toda. Essa estratégia visa reduzir os determinantes de base da saúde-doença e desse modo reduzir a incidência e morbimortalidade do agravo na população. As vantagens dessa estratégia são notáveis: ela é radical e poderosa, porque uma pequena mudança na distribuição do risco na população toda tem um grande efeito nos adoecimentos e mortes a ele associados. Várias medidas preventivas nessa estratégia já existem: uso de cinto de segurança nos automóveis, restrição do sal nos alimentos (em alguns países), proibição de propaganda do tabaco e de fumar nos espaços públicos fechados, inclusão de iodo no sal de cozinha, etc. Todavia, muitas outras mais seriam importantes, por exemplo: subsídios à produção de alimentos sem agrotóxicos, maior priorização de mobilidade sustentável nas cidades e diminuição das desigualdades sociais e econômicas, estas últimas um importante determinante da saúde-doença. ${ }^{28,29}$

No mesmo livro, Rose ${ }^{9}$ esboçou, sem desenvolver, as ideias de prevenção redutiva e aditiva. As medidas preventivas redutivas visam reduzir um risco aumentado já existente, visando restabelecer condições e modos de viver normais ou salutogênicos. Já as medidas preventivas aditivas são a introdução de um fator estranho, artificial ao corpo ou ao ambiente, para conferir proteção específica. O autor usou essa categorização para destacar a preferência pela abordagem populacional com medidas preventivas redutivas, pois são seguras e benéficas, ao reduzirem fatores sabidamente patogênicos e artificiais, aumentados com a industrialização, urbanização e o modo de vida ocidental moderno. Alógica dessas medidas é o "restabelecimento de condições ambientais e sociais e modos de viver favoráveis à saúde" (p. 4);9 o que se aproxima muito da PS.

Por outro lado, as medidas preventivas aditivas têm alto potencial de risco, não podem ser consideradas seguras e requerem rigorosa avaliação científica de sua eficácia e segurança. Elas somente devem ser consideradas para aplicação em populações ou indivíduos após consistentes e convergentes evidências de que o balanço benefícios $x$ danos é amplamente favorável. Ocorre que várias dessas medidas já foram introduzidas e viraram rotina, e novas vem sendo inseridas na APS e na saúde pública, tanto na abordagem de alto risco quanto na populacional. Elas não devem ser consideradas seguras porque foram implantadas. Sua manutenção deve ser sustentada por evidências atualizadas e também por dados epidemiológicos populacionais e estudos observacionais, pois o que foi implantado pode amanhã se mostrar danoso e ou ineficaz. Por isso, a P4 implica prestar redobrada atenção às ações preventivas aditivas, com uma postura cética e crítica continuada, especialmente em abordagem de alto risco, pelo seu pouco impacto positivo na morbimortalidade coletiva ${ }^{8} \mathrm{e}$ 
sua maior medicalização e potencial de danos. ${ }^{7}$ Convergente com Rose, ${ }^{9}$ a P4 implica uma preferência relativa por medidas preventivas redutivas em abordagem populacional, seguras e de maior impacto.

\section{Promoção da saúde e prevenção redutiva}

Na PS há ênfase e valorização de abordagens dos determinantes sociais e gerais do processo saúdedoença e, portanto, ações de amplo espectro voltadas à sociedade e à população. Com isso se visa construir sociedades e cidades saudáveis, prover e garantir salubridade, democracia e segurança para todos, além de resgatar e reforçar laços sociais de solidariedade e apoio social, sabidamente importantes. Por outro lado, como vimos, há quem enfatize a abordagem dos indivíduos, para que adotem estilos de vida saudáveis. ${ }^{30}$

As ações de PS na esfera societal e cultural convergem e até mesmo se identificam com várias medidas redutivas em abordagem populacional acima mencionadas. Seja pela via da criação de políticas, valores e ambientes saudáveis e do estímulo à participação e ao empoderamento psicológico e social (PS), seja pela via da redução dos riscos artificiais gerados pela sociedade moderna (prevenção redutiva), é evidente a convergência conceitual e operacional da PS com medidas preventivas redutivas.

Chor e Faerstein, ${ }^{31}$ também reconheceram a afinidade das ideias de Rose $^{9} \mathrm{com}$ a PS, mas o fizeram no sentido da convergência da estratégia de abordagem populacional com a PS. Adicionamos a esta convergência, intensificando-a, a preferência relativa da P4 pela prevenção redutiva em articulação com a abordagem populacional.

\section{Prevenção quaternária, prevenção redutiva e promoção da saúde}

Vimos acima que a P4 na prevenção orienta valorizar as medidas preventivas redutivas. Essas, por sua vez, convergem e em parte se identificam com ações de PS. Por isso, podemos afirmar que a P4 tem uma relação clara, embora indireta, de convergência com a PS. A categorização de Rose ${ }^{9}$ facilita perceber essa convergência e orientar ações de gestores e profissionais da APS para preferir relativamente medidas preventivas redutivas e avaliar criticamente ações preventivas aditivas, que merecem a aplicação do princípio da precaução. Tal princípio enfatiza que em caso de dúvida quanto ao balanço danos $\mathrm{x}$ benefícios, tais ações não devem ser implantadas ou devem ser suspensas se já em vigor. ${ }^{10}$

O conjunto das relações apresentadas está ilustrado na figura abaixo.

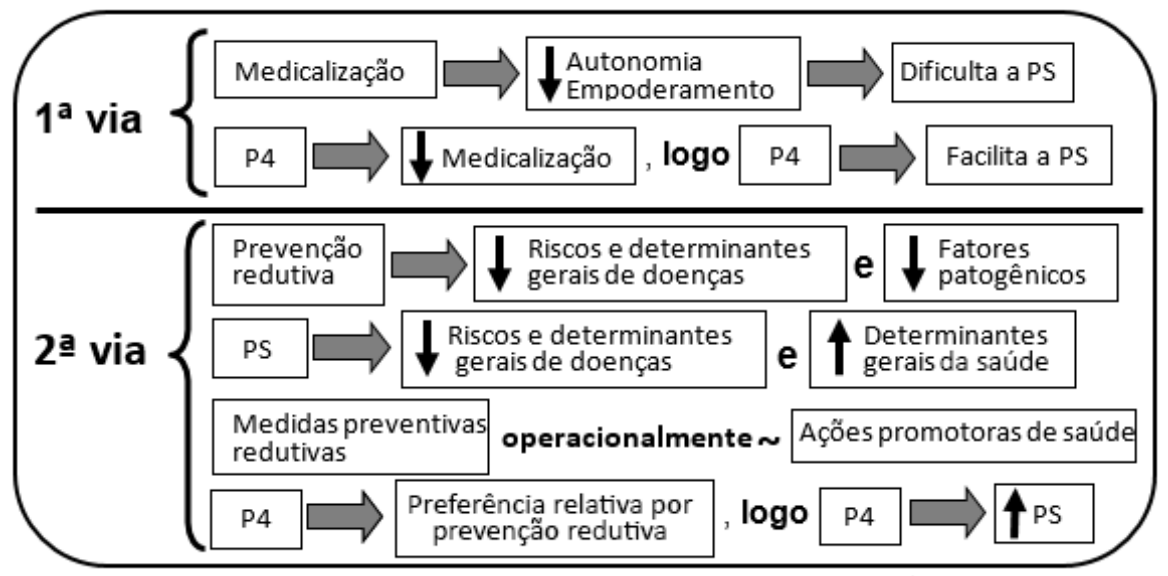

Legenda: PS = Promoção da saúde; P4 = Prevenção quaternária; $\sim=$ Similar; $\Rightarrow=$ Produz; $\boldsymbol{\uparrow}=$ Aumenta; $\downarrow$ = Diminui.

Fonte: Elaboração do autor. 


\section{CONSIDERAÇÕES FINAIS}

Embora tenham origens, focos e matrizes conceituais próprias e distintas, a P4 e a PS são convergentes. Em dimensão individual e grupal, reduzir danos iatrogênicos e a medicalização na APS abre espaço para a PS, demanda habilidades com ela convergentes e induz diálogos e iniciativas que dela se aproximam, que podem até se confundir operacionalmente e conceitualmente com PS.

Em dimensão coletiva e societal, a P4 contribui e se identifica operacionalmente com a PS na valorização das ações preventivas redutivas, sobretudo em abordagem populacional, que abordam, como a PS, determinantes sociais da saúde-doença, visando impactá-los positivamente.

A P4 é convergente com a PS nos serviços de saúde da APS em dimensão individual e comunitária e em dimensão macrossocial de políticas públicas. A clareza sobre essas relações facilita a compreensão da interface da P4 com a PS, bem como destaca a relevância da P4 no cuidado clínico individual e em ações coletivas na APS. As convergências aqui apresentadas provavelmente não esgotam as relações entre P4 e PS, mas facilitam a exploração teórica e prática de ambas e sua potencial sinergia.

\section{REFERÊNCIAS}

1. Bentzen N. WONCA International Dictionary of General/Family Practice. Copenhagen: Maanedskift Lager;2003.

2. Starfield B, Hyde J, Gérvas J, Heath I. The concept of prevention: a good idea gone astray?. J Epidemiol Community Health. 2008;62(7):58083. DOI: https://doi.org/10.1136/jech.2007.071027

3. Jamoulle M. Quaternary prevention, an answer of family doctors to overmedicalization. Int J Health Policy. 2015 Fev;4(2):61-4. DOI: https://doi.org/10.15171/ijhpm.2015.24

4. Depallens MA, Guimarães JMM, Almeida Filho N. Prevenção quaternária: um conceito relevante para a saúde pública? Uma análise bibliométrica e descritiva. Cad Saúde Pública. 2020;36(7):e00231819. DOI: http://dx.doi.org/10.1590/0102-311x00231819

5. Carta de Ottawa. In: Primeira Conferência Internacional sobre Promoção da Saúde [Internet]. Ottawa, Canadá; 1986; [acesso em 2019 Dez 30]. Disponível em: http://bvsms.saude.gov.br/bvs/publicacoes/carta_ottawa.pdf

6. Jamoulle M, Roland M, Bae J, Heleno B, Visentin G, Gusso GDF, et al. Ethical, pedagogical, socio-political and anthropological implications of quaternary prevention. Rev Bras Med Fam Comunidade. 2018 Jan;13(40):1-14.

7. Fortune K, Becerra-Posada F, Buss PM, Galvão LA, Contreras A, Murphy M, et al. Health promotion and the agenda for sustainable development, WHO Region of the Americas. Bull WHO. 2018;96:621-6.

8. Conrad P.The medicalization of society: on the transformation of human coditions into treatable disorders. Baltimore: The Johns Hopkins University Press; 2007.

9. Rose G. Estratégias da medicina preventiva. Porto Alegre: Artmed; 2010.

10. Tesser CD, Norman AH. Geoffrey Rose e o princípio da precaução: para construir a prevenção quaternária na prevenção. Interface (Botucatu). 2019;23:e180435. DOI: http://dx.doi.org/10.1590/interface.180435

11. Rose N. Beyond medicalisation. Lancet. 2007;369:700-1.

12. Clarke AE, Mamo L, Fosket JR, Fishman JR, Shim JK. Biomedicalization: technoscience, health, and illness in the U.S. Durhan, NC: Duke University Press; 2010.

13. Petryna A. Biological citizenship after Chernobyl. In: Hahn RA, Inborn M, eds. Anthropology and Public Health: bridging differences in culture and society. New York: Oxford University Press; 2009. p. 623-51.

14. Illich I. A expropriação da saúde: nêmesis da medicina. São Paulo: Nova Fronteira; 1975.

15. Canguilhem G. O normal e o patológico. Rio de Janeiro: Forense-Universitária; 2002.

16. Martins C, Godycki-Cwirko M, Heleno B, Brodersen J. Quaternary prevention: reviewing the concept. Eur J Gen Pract. 2018 Dez;24(1):106-11.

17. Norman AH, Tesser CD. Quaternary prevention: a balanced approach to demedicalisation. Br J Gen Pract. 2019;69(678):28-9. DOI: https://doi.org/10.3399/bjgp19X700517 
18. Leavell H, Clark EG. Medicina preventiva. São Paulo: McGraw-Hill; 1976.

19. Arouca S. O dilema preventivista. São Paulo: Editora UNESP; 2003.

20. Tesser $\mathrm{CD}$, Norman AH. Differentiating clinical care from disease prevention: a prerequisite for practicing quaternary prevention. Cad Saúde Pública. 2016;32(10):e00012316. DOI: http://dx.doi.org/10.1590/0102-311X00012316

21.Prado NMBL, Santos AM. Promoção da saúde na Atenção Primária à Saúde: sistematização de desafios e estratégias intersetoriais. Saúde Debate. 2018 Set;42(spe 1):379-95. DOI: http://dx.doi.org/10.1590/0103-11042018s126

22. Carvalho SR. Os múltiplos sentidos da categoria "empowerment" no projeto de Promoção à Saúde. Cad Saúde Pública. 2004 Jul/ Ago;20(4):1088-95. DOI: http://dx.doi.org/10.1590/S0102-311X2004000400024

23. Carvalho SR, Gastaldo D. Promoção à saúde e empoderamento: uma reflexão a partir das perspectivas crítico-social pós-estruturalista. Ciênc Saúde Coletiva. 2008;13(Supl 2):2029-40. DOI: http://dx.doi.org/10.1590/S1413-81232008000900007

24. Tesser CD. Introdução. In: Tesser CD, org. Medicalização social e atenção à saúde no SUS. São Paulo: Hucitec; 2010. p.11-33.

25. Camargo Junior KR. Biomedicina, ciência e saber: uma abordagem crítica. São Paulo: Hucitec; 2003.

26. Castiel LD, Álvarez-Dardet C. A saúde persecutória: os limites da responsabilidade. Rio de Janeiro: Editora Fiocruz; 2007.

27. Tesser CD. Três considerações sobre a "má medicina". Interface (Botucatu). 2009;13(31):273-86.

28. Wilkinson RG, Pickett KE. The spirit level: why more equal societies almost always do better. London: Penguim; 2009.

29. Pickett KE, Wilkinson RG. Income inequality and health: a causal review. Soc Sci Med.2015 Mar;128:316-26. DOI:https://doi.org/10.1016/j. socscimed.2014.12.031

30. Sutherland RW, Fulton MJ. Health promotion. In: Sutherland RW, Fulton MJ, eds. Health care in Canada. Ottawa: Canadian Public Health Association (CPHA); 1992. p.161-81.

31. Chor D, Faerstein E. Um enfoque epidemiológico da promoção da saúde: as idéias de Geoffrey Rose. Cad Saúde Pública. 2000 Jan;16(1):241-4. DOI: http://dx.doi.org/10.1590/S0102-311X2000000100025 